\title{
Uso da Ciprofloxacina durante a Prenhez de Ratas: Efeitos sobre a Mãe e Fetos
}

\author{
Use of Ciprofloxacin in Pregnant Rats: Effects on Mother and Fetuses
}

\begin{abstract}
Mário Silva Approbato, Racine Procópio Teixeira, Kátia Karina Verolli Moura, Cassiano Rodrigues Isaac, Klayvison Koslyk Alves, Fabiana Nunes de Carvalho, Mauri Felix de Souza
\end{abstract}

\begin{abstract}
RESUM0
Objetivos: estudar os efeitos da ciprofloxacina sobre a taxa de abortos, ganho de peso durante a gravidez, morte materna e fetal, malformações fetais grosseiras, número de recém-nascidos, peso dos filhotes e análise dos reflexos neurológicos dos filhotes.

Métodos: foram utilizadas 30 ratas da linhagem Wistar, divididas em três grupos. Os grupos tratados receberam ciprofloxacina e o grupo controle soro fisiológico, uma vez ao dia, durante os primeiros 7 dias após o cruzamento. As doses para os grupos D50 e D100 foram, respectivamente, de 50 e $100 \mathrm{mg}$ de ciprofloxacina por $\mathrm{kg}$ de peso corporal por dia. Os parâmetros avaliados foram: o ganho de peso durante a prenhez, a prevalência de abortamentos, mortes fetal e materna e malformações fetais grosseiras. Nos 217 filhotes nascidos analisamos o número, o peso e reflexos neurológicos (postural, preensão e orientação) no $1^{\circ}, 3^{\circ}, 5^{\circ}$ e $10^{\circ}$ dia de vida por ninhada.

Resultados: não houve diferenças estatisticamente significativas no número de ratas que ficaram prenhes, na média de ganho de peso materno ou número de filhotes. Os filhotes apresentaram diferenças significativas nos pesos das observações do $3^{\circ}, 5^{\circ}$ e $10^{\circ}$ dia ( $p=0,006,0,01$ e 0,03, respectivamente), sendo a média menor no grupo D100. Observamos alteração significativa ( $p$ $=0$,002) na positividade do reflexo de orientação no primeiro dia de vida, alteração que não se manteve nas outras observações. Não se observou caso de abortamento ou malformações grosseiras nos recém-nascidos.

Conclusões: a ciprofloxacina alterou o peso ao nascimento e o reflexo dos filhotes nos primeiros dias de vida. Portanto, deve-se estudar a restrição ao seu uso durante a gravidez.
\end{abstract}

PALAVRAS-CHAVE: Antibióticos. Drogas na gravidez. Malformações fetais. Infecção urinária.

Introdução

A ciprofloxacina é uma fluorquinolona de segunda geração. As quinolonas são mais utilizadas nas infecções dos trato urinário (ITU), pois atingem maior concentração nos túbulos renais ${ }^{1}$. Essas infecções ocorrem mais freqüentemente em mulheres, sendo mais comuns nas sexualmente ativas, pertencentes às classes sociais menos privilegiadas e na faixa dos $30 \operatorname{anos}^{2}$. A

$\overline{\text { Laboratório de Reprodução - Hospital das Clínicas - FM - }}$ Universidade Federal de Goiás

Correspondência:

Mário Silva Approbato

Av. $5^{a}$ Radial, 131/ 103 - Setor Pedro Ludovico

74823-030 - Goiânia - GO

email: approbat@medicina.ufg.br
ITU é complicação médica comum da gestação ${ }^{3}$ e é devida às mudanças anatômicas e fisiológicas que ocorrem no sistema urinário durante este período.

A bacteriúria gestacional tende a ser persistente, tem um baixo índice de cura espontânea e se não tratada pode evoluir até pielonefrite aguda $^{4}$. Vários autores relatam que, devido ao risco de bacteriúria assintomática, as gestantes devem ser rastreadas em sua primeira avaliação pré-natal ${ }^{4}$.

Esta bacteriúria geralmente aparece pela primeira vez antes da décima segunda semana de gestação ${ }^{5}$. Uma vez detectada, deve ser tratada, em decorrência do risco do desenvolvimento de pielonefrite. 
Linseman et al. ${ }^{6}$ trataram camundongos com ciprofloxacina, 50 e $200 \mathrm{mg} / \mathrm{kg} /$ dia, e ácido pipemídico, 50,400 e $3.150 \mathrm{mg} / \mathrm{kg} / \mathrm{dia}$. Os grupos tratados na dose de $200 \mathrm{mg} / \mathrm{kg} /$ dia durante 5 dias ou com ácido pipemídico (400 e 3.150 $\mathrm{mg} / \mathrm{kg} /$ dia) durante 7 dias tiveram maior incidência de lesões articulares como perda dos condrócitos, degeneração da matriz e erosão da cartilagem articular que os camundongos tratados com ácido pipemídico (400 mg/ $\mathrm{kg} / \mathrm{dia}) \mathrm{du}-$ rante 14 dias, sugerindo a possibilidade de uma reversibilidade das lesões à medida que o tempo de tratamento se prolonga.

Foi então realizado este estudo, em decorrência da possibilidade do uso da ciprofloxacina no período inicial de gravidez (quando a paciente ainda não percebe que está grávida) e dos relatos de seus efeitos sobre a gestação.

\section{Material e Métodos}

Esse foi um estudo experimental, prospectivo e controlado, sendo utilizadas 30 ratas, da linhagem Wistar, com idade inicial entre 4 a 6 meses, peso médio de 214,47 g, divididas em 3 grupos: 10 ratas (designado grupo tratado D50) receberam ciprofloxacina $50 \mathrm{mg} / \mathrm{kg}$ e 10 (grupo tratado D100) receberam ciprofloxacina $100 \mathrm{mg} /$ $\mathrm{kg}$, diluída em soro fisiológico. As doses utilizadas nesse trabalho foram semelhantes às utilizadas por Linseman et al. ${ }^{6}$. Dez ratas (grupo controle C) receberam soro fisiológico pela mesma via. O período de uso foi de sete dias, com início no $1^{\circ}$ dia após o fim do cruzamento, uma vez ao dia, por via oral por meio de sonda. As ratas foram mantidas em ciclo de luz de 12 horas, em caixas separadas. A ração fornecida foi de 15-20 $\mathrm{g} / \mathrm{rata} /$ dia e água ad libitum.

Os parâmetros estudados foram: porcentagem de abortos, ganho de peso durante a gravidez (as ratas foram pesadas semanalmente no $1^{\circ}, 7^{\circ}, 14^{\circ}$ e $21^{\circ}$ dia até o parto em balança Micronal com precisão de 0,01 g), morte materna e fetal, malformações fetais grosseiras, número de recém-nascidos, peso dos filhotes no $1^{\circ}$, 3으, 5 e 10ำ dia de vida e nos mesmos dias análise dos reflexos neurológicos dos filhotes: postural (os filhotes foram colocados em decúbito dorsal, sendo considerado reflexo positivo quando reassumiam a posição de decúbito ventral), preensão (um instrumento de ponta foi encostado na planta das patas, sendo considerado positivo quando havia preensão do instrumento) e orientação (o filhote era suspenso pela cauda no espaço, próximo a uma mesa). Foram considerados positivos aqueles que se orientavam para alcançar a mesa.

Para o ganho de peso materno e dos filhotes, foi utilizada análise de variância. Para a análise dos reflexos neurológicos foi utilizado o teste do $\chi^{2}$. Para as malformações foram utilizados métodos descritivos. Para os cálculos foi utilizado o pacote de estatística SPSS versão 7.5.

\section{Resultados}

Nasceram 217 filhotes dos três grupos, que foram avaliados até o $10^{\circ}$ dia pós-parto. Houve gestação em $90 \%$ do grupo controle, $70 \%$ do grupo tratado D50 e $80 \%$ do grupo tratado D100, diferença que não foi significativa $(\mathrm{p}=\mathrm{ns})$.

Em relação ao peso materno, observamos na primeira avaliação após o início do tratamento ( $7^{\circ}$ dia de gravidez) que as médias dos grupos tratado D100, tratado D50 e controle foram, respectivamente, 202,6 g, 222,3 g e 202,3 g, diferença estatisticamente não-significante. Na segunda avaliação após o início do tratamento ( $14^{\circ}$ dia de gravidez), as médias de peso materno dos grupos tratado D100, tratado D50 e controle foram, respectivamente, $221,3 \mathrm{~g}, 232,6 \mathrm{~g}$ e 212,0 g, diferença também estatisticamente não-significativa $(\mathrm{p}=\mathrm{ns})$. Na terceira avaliação após o início do tratamento (21ํ dia de gravidez), as médias de peso materno dos grupos tratado D100, tratado D50 e controle foram, respectivamente, $249,1 \mathrm{~g}, 266,4$ g e $260,5 \mathrm{~g}(\mathrm{p}=\mathrm{ns})$.

Quanto ao número de recém-nascidos por rata, obtivemos as seguintes medianas: no grupo tratado D100, 9,5, no grupo D50, 9,0 e no grupo controle, 10 ( $\mathrm{p}=\mathrm{ns})$.

Quanto ao peso dos recém-nascidos no primeiro dia de vida, as médias nos grupos tratado D100, tratado D50 e controle foram, respectivamente, $5,4 \mathrm{~g}, 5,7 \mathrm{~g}$ e $5,5 \mathrm{~g}(\mathrm{p}=\mathrm{ns})$. No terceiro dia de vida, a média de peso nos grupos tratado D100, tratado D50 e controle foram, respectivamente, 6,5 g, 6,9 g e 6,6 g, diferença significante $(p=0,006)$. No quinto dia de vida, a média de peso nos grupos tratado D100, tratado D50 e controle foram, respectivamente, 8,2 g, 8,9 g e $8,3 \mathrm{~g}$, diferença estatisticamente significativa $(\mathrm{p}=0,01)$. No décimo dia de vida, a média do peso nos grupos tratado D100, tratado D50 e controle foram, respectivamente, $13,1 \mathrm{~g}, 14,3 \mathrm{~g}$ e $13,7 \mathrm{~g}$, diferença estatisticamente significativa $(p=0,03)$, Figura 1 . 


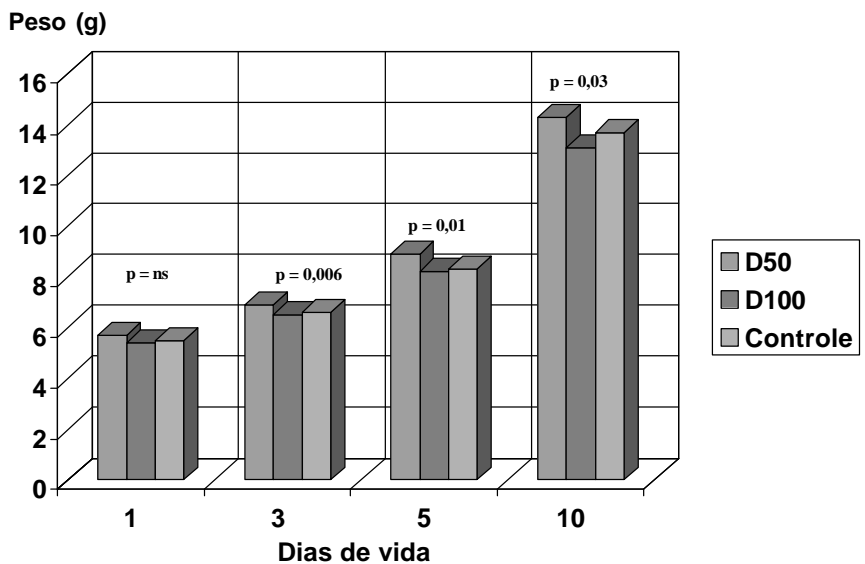

Figura 1 - Distribuição de filhotes segundo o ganho de peso corporal até $010^{\circ}$ dia e a dose de ciprofloxacina empregada durante a prenhez. D50 - $50 \mathrm{mg} / \mathrm{kg} / \mathrm{dia}, \mathrm{D} 100$ - $100 \mathrm{mg} / \mathrm{kg} / \mathrm{dia}$, controle - soro fisiológico. ns, Não-significante.

A respeito dos reflexos neurológicos dos recém-nascidos, observamos que no primeiro dia de vida houve positividade do reflexo postural no grupo tratado D100 em 96,2\%, no grupo tratado D50 em $100 \%$ e no grupo controle em $100 \%$, não sendo essa diferença significante. No terceiro dia de vida houve positividade no grupo tratado D100 em 96,1\%, no grupo tratado D50 em $98,2 \%$ e no grupo controle em $92 \%(p=n s)$. No quinto dia de vida, houve positividade no grupo tratado D100 em 100\%, no grupo tratado D50 em $100 \%$ e no grupo controle em $98,7 \%(\mathrm{p}=\mathrm{ns})$. No décimo dia de vida, houve positividade no grupo tratado D100 em $100 \%$, no grupo tratado D50 em $100 \%$ e no grupo controle em 98,6\% ( $\mathrm{p}=\mathrm{ns}$ ). Não observamos alteração do reflexo de preensão dos animais tratados com ciprofloxacina e animais controle nos dias de avaliação (1으, $3^{\circ}, 5^{\circ}$ e $10^{\circ}$ dia de vida). Com relação ao reflexo de orientação (Figura 2) encontramos no primeiro dia de vida uma positividade de $64,5 \%$ no grupo tratado D100, 54,3\% no grupo tratado D50 e $37,1 \%$ no grupo controle, diferença estatisticamente significativa $(p=0,002)$. No terceiro dia de vida, a positividade foi de $70,3 \%$ no grupo tratado D100, 56,1\% no grupo tratado D50 e $68 \%$ no grupo controle, $(\mathrm{p}=\mathrm{ns})$. No quinto dia de vida, a positividade foi de $71,4 \%$ no grupo tratado D100, 61,4\% no grupo tratado D50 e $64,9 \%$ no grupo controle ( $\mathrm{p}=\mathrm{ns})$. No décimo dia de vida, a positividade foi de $43,2 \%$ no grupo tratado D100, $72,7 \%$ no grupo tratado D50 e $56,6 \%$ no grupo controle ( $\mathrm{p}=\mathrm{ns})$.

Não foram observados abortos ou malformações grosseiras nos filhotes.

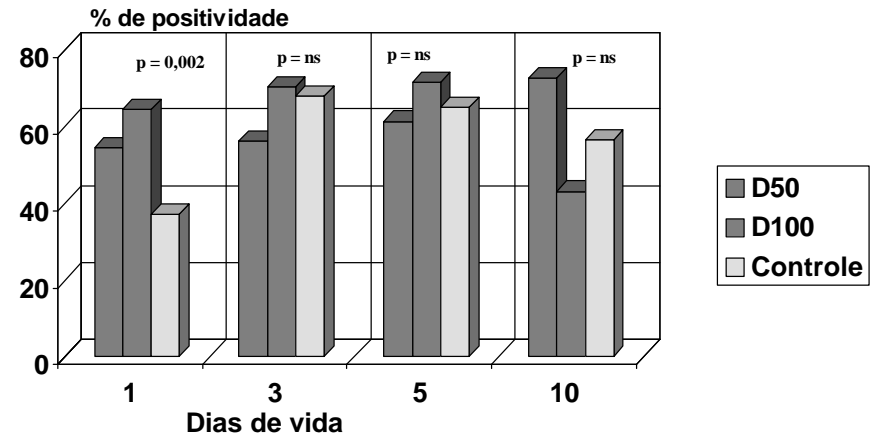

Figura 2 - Distribuição do número de filhotes segundo o reflexo de orientação e a dose de ciprofloxacina empregada durante a prenhez. D50 - $50 \mathrm{mg} / \mathrm{kg} / \mathrm{dia}$, D100 - $100 \mathrm{mg} / \mathrm{kg} / \mathrm{dia}$ e controle - soro fisiológico. ns, Não-significante.

\section{Discussão}

Não houve diferenças significativas em relação ao número de recém nascidos entre os grupos avaliados, mostrando que a fertilidade e a gestação das ratas não foram influenciadas pelo tratamento. Resultado semelhante foi encontrado por Watanabe et al. ${ }^{8}$ que estudaram estes efeitos usando respectivamente a lomefloxacina e a levofloxacina.

Os filhotes nasceram sem diferenças significativas com relação ao peso, apresentando entretanto, diferenças no ganho ponderal até o $10^{\circ}$ dia. Foi observado que os filhotes do grupo tratado D50 apresentaram o maior ganho ponderal até o $10^{\circ}$ dia, ao passo que os do grupo D100 apresentaram o menor. Estes resultados indicam que o aumento da dose de ciprofloxacina administrada às ratas durante a prenhez pode influenciar os primeiros dias do desenvolvimento dos filhotes. Estes resultados foram semelhantes aos encontrados no estudo desenvolvido por Watanabe et al. ${ }^{8}$, no qual não foram encontrados efeitos adversos no grupo tratado com levofloxacina na dose de $360 \mathrm{mg} / \mathrm{kg}$ e foram encontrados redução do peso dos filhotes e retardo da ossificação (este último parâmetro não foi analisado em nosso estudo) no grupo tratado com a dose de $810 \mathrm{mg} / \mathrm{kg}$.

Quanto ao reflexo de orientação, foi observado, no primeiro dia de vida dos filhotes, que os grupos tratados (principalmente D100) apresentaram maior positividade ao teste que o grupo controle. Esta alteração não permaneceu nas observações do $3^{\circ}$, $5^{\circ}$ e $10^{\circ}$ dia, indicando que a ciprofloxacina, na metodologia por nós utilizada, pode influenciar de maneira temporária o desenvolvimento neurológico dos filhotes. Tarantal et al. ${ }^{9}$ não encontraram alterações no desenvolvi- 
mento emocional e habilidade motora dos filhotes em seu estudo com prulifloxacina.

Não foram observados abortos nas gestações das ratas ou malformações grosseiras nos filhotes. Apesar de resultados em animais não poderem ser aplicados em seres humanos, efeitos similares foram encontrados por Berkovitch et al. ${ }^{10}$ em seu estudo com 38 mulheres tratadas com ciprofloxacina ou norfloxacina durante $o$ primeiro trimestre de gestação.

Estudos feitos na Universidade de Toronto testando uma quinolona criada recentemente e que ainda não está sendo usada no Brasil, a prulifloxacina, mostraram que essa droga não tem aparente efeito na função reprodutiva dos pais e no desenvolvimento dos fetos. Ela não influi no número dos nascidos vivos e na diferenciação, desenvolvimento funcional, emocional, habilidade motora e performance reprodutiva dos filhotes $^{11,12}$. Outros estudos feitos em coelhas mostraram que aquelas que receberam doses mais elevadas de prulifloxacina tiveram maior quantidade de fetos mortos ${ }^{13,14}$. Entretanto, estudando os efeitos da nova quinolona temafloxacina em macacas, não foram observadas diferenças significativas no crescimento dos fetos e embriões quando comparadas com o grupo controle ${ }^{9}$.

Este estudo mostrou que a ciprofloxacina tem efeitos na gravidez e portanto o seu uso deve ser restrito a situações em que, por ausência de outro medicamento eficaz e seguro, os riscos potenciais ao desenvolvimento do feto sejam menores que os benefícios do tratamento.

\section{SUMMARY}

Purpose: to study the effects of ciprofloxacin on abortion percentage, maternal weight gain during pregnancy, fetal and maternal death, gross fetal malformation, newborn number, weight and neurological reflexes.

Methods: we used 30 Wistar rats, divided into three groups: D50-treated (ciprofloxacin, $50 \mathrm{mg} / \mathrm{kg}$ ); D100-treated (ciprofloxacin $100 \mathrm{mg} / \mathrm{kg}$ ) and control group which received physiological saline per os, from the 1st to the 7th day after mating. We studied abortion percentage, maternal weight gain during pregnancy, fetal and maternal death, gross fetal malformation, newborn number and weight at 1 st, 3rd, 5th and 10th day of life and newborn neurological reflexes at $1 \mathrm{st}, 3 \mathrm{rd}$, 5th and 10th day of life.

Results: there was no difference between groups in the number of rats that became pregnant. The same was found for maternal weight gain and newborn number. There was a difference in newborn mean weight on day $3 \mathrm{rd}$, 5th and 10th $(p=0.006,0.01$ and 0.03 , respectively). The D100 newborn group was the one with less weight gain up to the 10th day of life. We found a significant difference ( $p=$ $0.002)$ in the newborn orientation reflex on the 1st day of life, that disappeared afterwards. No abortion or gross malformation was found in this study.

Conclusions: ciprofloxacin modified the newborn weight and reflex on the first days of life. In conclusion, we consider that the use of ciprofloxacin should be restricted during the pregnancy.

KEY WORDS: Antibiotics. Drugs in pregnancy. Fetal malformation. Urinary infection.

\section{Referências}

1. Mandell GL, Sande MA. Fármacos antimicrobianos. In: Gilman AG, Rall TW, Nies AS, Taylor P, editores. As Bases Farmacológicas da Terapêutica. $8^{\mathrm{a}}$ ed. Rio de Janeiro: Guanabara Koogan; 1991. p.700-1.

2. Toomey KE, Barnes RC. Treatment of Chlamydia trachomatis genital infection. Rev Infect Dis 1990; 12 Suppl 6:S645-55.

3. Pedler SJ, Orr KE. Infecções bacterianas fúngicas e parasitárias. In: William MB, Marshall DL, editores. Complicações Médicas na Gravidez. $1^{\text {a }}$ ed. Porto Alegre: Artes Médicas; 1993. p.285-9.

4. Lindheimer MD, Davison JM. Complicações renais. In: William MB, Marshall DL, editores. Complicações Médicas na Gravidez. $1^{\text {a }}$ ed. Porto Alegre: Artes Médicas; 1993. p.28-44.

5.Carvalho WA. Quinolonas. In: Silva P, editor. Farmacologia. $1^{\text {a }}$ ed. Rio de Janeiro: GuanabaraKoogan; 1994. p.1112-26.

6.Linseman DA, Hampton SA, Branstetter DG. Quinolone-induced arthropathy in the neonatal mouse. Morphological analysis of articular lesions produced by pipemidic acid and ciprofloxacin. Fundam Appl Toxicol 1995; 28:5964.

7. Snedecor GW, Cochran WG. Statistical methods. $6^{\mathrm{a}}$. ed. Ames: The Iowa State University Press; 1967. p. 120.

8. Watanabe T, Fujikawa K, Harada S, Okura K, Sasaki $\mathrm{T}$, Takaxama S. Reproductive toxicity of the new quinolone antibacterial agent levofloxacin in rats and rabbits. Arzneimittelforschung 1992; 43:3747.

9. Tarantal AF, Lehrer SB, Lasley BL, Hendrickx AG. Developmental toxicity of temafloxacin hydrochloride in the long-tailed macaque (Macaca faciculares). Teratology 1990; 42:233-42. 
10.Berkovitch M, Pastuszak A, Gazarian M, Lewis M, Koren G. Safety of the new quinolones in pregnancy. Obstet Gynecol 1994; 84:535-8.

11.Morinaga T, Fujii S, Furukawa S, et al. Reproductive and developmental toxicity studies of prulifloxacin (NM441)(1). A fertility study in rats by oral administration. J Toxicol Sci 1996; 21 Suppl 1:171-85.

12.Morinaga T, Fujii S, Furukawa S, et al. Reproductive and developmental toxicity studies of prulifloxacin (NM441)(2). A teratogenicity study in rats by oral administration. J Toxicol Sci 1996; 21 Suppl 1:187-206.

13.Morinaga T, Fujii S, Furukawa S, et al. Reproductive and developmental toxicity studies of prulifloxacin (NM441)(3). A teratogenicity study in rabbits by oral adminstration. J Toxicol Sci 1996; 21 Suppl 1:207-17.

14.Morinaga T, Fujii S, Okazaki I, et al. Reproductive and developmental toxicity studies of prulifloxacin (NM441)(4). A perinatal and postnatal study in rats by oral administraction. J Toxicol Sci 1996; 21 Suppl 1:219-30.

\title{
COMUNICAD0
}

\section{Desde 0 dia 20 de novembro a Secretaria da FEBRASGO está funcionando em novo endereço:}

\author{
Av. das Américas, 8445 sala 711 - Barra da Tijuca \\ Rio de Janeiro - RJ - CEP 22793-081 \\ Tel: (21) 487-6336 Fax: (21) 429-5133 \\ e-mail: secretaria_executiva@ febrasgo.org.br
}

